\title{
Afp::mCherry, a red fluorescent transgenic reporter of the mouse visceral endoderm
}

\author{
Manuel Viotti ${ }^{1,2}$, Sonja Nowotschin ${ }^{1}$, and Anna-Katerina Hadjantonakis ${ }^{1, \#}$ \\ ${ }^{1}$ Developmental Biology Program, Sloan-Kettering Institute, New York, NY, USA. \\ ${ }^{2}$ Biochemistry, Cell and Molecular Biology Graduate Program, Weill Medical College of Cornell \\ University, New York, NY, USA.
}

\section{SUMMARY}

Live imaging of genetically-encoded fluorescent protein reporters is increasingly being used to investigate details of the cellular behaviors that underlie the large-scale tissue rearrangements that shape the embryo. However, the majority of mouse fluorescent reporter strains are based on the green fluorescent protein (GFP). Mouse reporter strains expressing fluorescent colors other than GFP are therefore valuable for co-visualization studies with GFP, where relative positioning and relationship between two different tissues or compartments within cells is being investigated. Here, we report the generation and characterization of a transgenic Afp::mCherry mouse strain in which cis-regulatory elements from the Alpha-fetoprotein ( $A f p$ ) locus were used to drive expression of the monomeric Cherry red fluorescent protein. The Afp:: $m$ Cherry transgene iss based on, and recapitulates reporter expression of a previously described Afp::GFP strain. However we note that perdurance of mCherry protein is not as prolonged as GFP, making the Afp::mCherry line a more faithful reporter of endogenous Afp expression. Afp::mCherry transgenic mice expressed mCherry specifically in the visceral endoderm and its derivatives, including the visceral yolk sac, gut endoderm, fetal liver and pancreas of the embryo. The Afp::mCherry reporter was also noted to be expressed in other documented sites of $A f p$ expression including hepatocytes, as well as in pancreas, digestive tract and brain of postnatal mice.

\section{Keywords}

mouse embryo; visceral endoderm; endoderm morphogenesis; yolk sac; liver; intestine; alphafetoprotein; live imaging; confocal; fluorescent protein; mCherry

\begin{abstract}
Macroscopic changes in tissue or organ structure result from coordinated changes in the arrangements and shapes of cells. To this end, live imaging of genetically-encoded fluorescent protein reporters is increasingly being used to investigate details of the cellular behaviors that underlie the large-scale tissue rearrangements that shape the embryo. The cell movements underlying morphogenesis of the early mouse embryo are complex and not well understood. Gastrulation is the event that results in the formation of the three germ layers from the pluripotent epiblast and leads to the elaboration of the embryonic axes. Prior to the initiation of gastrulation, the mouse embryo comprises a bilaminar cup-shaped structure (often referred to as the egg cylinder stage) consisting of visceral endoderm, an epithelium that encapsulates the extraembryonic ectoderm proximally, and the epiblast distally (Nowotschin and Hadjantonakis, 2010). The proximal visceral endoderm overlying the extraembryonic ectoderm has generally been referred to as the exVE, while the proximally
\end{abstract}

\footnotetext{
\#Author for correspondence hadj@mskcc.org 212-639-3159 (phone) 646-422-2355 (FAX).
} 
positioned visceral endoderm overlying the epiblast has been referred to as the emVE (Mesnard et al., 2006). The visceral endoderm, which has long been recognized as critical for nutrient uptake and transport, also plays a role in the morphogenesis and patterning of the epiblast (Srinivas, 2006).

Using a transgenic strain of mice in which we placed a green fluorescent protein (GFP) reporter under the transcriptional control of the enhancer/promoter sequences from the Alpha-fetoprotein ( $A f p$ ) gene, we could label the visceral endoderm of the early postimplantation embryo as well as many of its derivatives, including the visceral yolk sac (Kwon et al., 2006). Furthermore, we noted that when compared to other cis-regulatory elements driving visceral endoderm-specific transgene expression, for example from the Transthyretin (Ttr) locus, Afp provided more robust levels of reporter expression (Kwon and Hadjantonakis, 2009).

As a consequence we noted the perdurance of GFP protein in cells of the visceral endoderm overlying the epiblast (often referred to as emVE) (Mesnard et al., 2006; Nowotschin and Hadjantonakis, 2010) that would otherwise have downregulated transcription of many visceral endoderm markers including Afp and Ttr (Kwon et al., 2008). The Afp::GFP strain therefore served as a short-term visceral endoderm lineage tracer. 3D time-lapse analyses of the GFP-positive visceral endoderm in Afp::GFP embryos revealed the widespread dispersal of the emVE epithelium during gastrulation. We hypothesized that visceral endoderm dispersal was achieved through widespread intercalation of epiblast cells into the preexisting visceral endoderm epithelium (Ferrer-Vaquer et al., 2010; Kwon et al., 2008; Nowotschin and Hadjantonakis, 2010). These genetic-labeling and live imaging studies have led to a revised model of mouse gut endoderm morphogenesis, in which epiblast-derived cells egress multi-focally into the emVE epithelial layer and in doing so disperse emVE cells, rather than displacing the VE en masse (Kwon et al., 2008). Furthermore, the observation that in Afp::GFP transgenic embryos GFP-positive emVE cells remained associated with the epiblast, and at later stages they or their descendents were detected within the gut tube, suggested that at least some of the cells comprising the emVE, a lineage previously believed to be exclusively extraembryonic, might contribute to the embryoproper, thereby challenge the notion of a strict segregation between extraembryonic and embryonic tissue lineages in mammals (Kwon et al., 2008).

The majority of mouse fluorescent reporter strains are based on GFP. Originally isolated from the jellyfish Aequorea victoria, this fluorescent protein is the most widely used reporter across model systems. GFP has been extensively engineered and mutated to obtain variants exhibiting improved photostability and brightness, such as EGFP, as well as spectrally-distinct variants that are amenable to use in mice (Nowotschin et al., 2009b). Mouse reporter strains expressing fluorescent colors other than GFP are valuable for covisualization studies with GFP, where relative positioning and relationship between two different tissues or compartments within cells is being investigated (Nowotschin $e t$ al., 2009a). In addition, due to their longer wavelength excitation and emission spectra, red fluorescent proteins are favorable for live imaging studies because of their reduced cellular toxicity. There is therefore a growing need for the development of mouse live imaging reporter strains in colors other than green.

We recently generated a Ttr::RFP strain, in which the red fluorescent protein mRFP1 (Campbell et al., 2002) was expressed under the control of the Ttr promoter (Kwon and Hadjantonakis, 2009). In this reporter strain mRFP1 labeled the entire visceral endoderm. However, since comparative reporter experiments suggested that the Ttr cis-regulatory elements do not drive transgene expression as robustly as those from the $A f p$ locus (Kwon and Hadjantonakis, 2009), we wanted to develop spectral-variant reporter strains using the 
Afp cis-regulatory elements. The weak expression of the Ttr::RFP coupled with the increasing necessity for labeling tissues such as the visceral endoderm in co-visualization experiments with other GFP-based reporter mouse lines led us to generate a new transgenic mouse strain expressing the monomeric red fluorescent protein mCherry (Shaner et al., 2004), under the Afp cis-regulatory elements. Due to its fast maturation, brightness and high photostability, mCherry has already been shown to be a suitable RFP for co-visualization live imaging experiments ex vivo in mouse embryonic stem (ES) cells, and in vivo in both mouse embryo chimeras comprising mCherry expressing ES (Nowotschin et al., 2009a), and in transgenic mice (Fink et al., 2010).

Here we report the generation of a transgenic Afp::mCherry mouse strain that expresses the monomeric red fluorescent protein mCherry specifically in the visceral endoderm and its derivatives, the visceral yolk sac and gut endoderm, as well as in the fetal liver and pancreas. In addition, we show the Afp::mCherry reporter exhibits mCherry expression in hepatocytes, as well as in liver, pancreas, digestive tract and brain of postnatal mice. Our analyses confirm that generally the mCherry reporter in the Afp::mCherry strain is expressed in an equivalent spatio-temporal pattern as the GFP reporter in the Afp::GFP strain, with slightly reduced reporter perdurance. By co-visualization we demonstrate the utility of Afp::mCherry reporter as a suitable alternative to the $A f p:: G F P$ reporter in experiments where it is necessary to label visceral endoderm cells in a color other than green, thereby making combinatorial reporter visualization studies now possible.

\section{Generation of an Afp::mCherry mouse strain}

The $p A f p:: m$ Cherry construct design was based on our previously reported $p A f p:: G F P$ construct (Kwon et al., 2006). Having generated a pAfp::mCherry construct we confirmed robust reporter expression as determined by red fluorescence in Afp-expressing cell lines (Fig. 1). Transient transfection of mouse and human hepatocytes (Hepa1-6 and HepG2, respectively) and a human colorectal adenocarcinoma cell lines (Caco-2) revealed robust red fluorescence suggesting that the pAfp::mCherry construct was functional (Fig. 1b). $p A f p:: G F P$ and $p A f p:: m C h e r r y$ were compared and shown to exhibit similar expression in this assay.

Having validated the $p A f p:: m$ Cherry construct in cells, we proceeded to generate the mouse transgenic stain. We identified three transgene-containing founder $\left(\mathrm{F}_{0}\right)$ animals. To validate correct expression of the mCherry reporter in the visceral endoderm, Afp::mCherry founders were crossed to wild-type animals and late streak (LS) to early headfold (EHF) stage hemizygous transgenic embryos, stages where we expected to observe robust and localized reporter expression, were recovered and screened for red fluorescence. We identified one founder that produced embryos displaying more robust red fluorescence within and throughout the visceral endoderm, and so focused our analyses on this line. We generated $\mathrm{F}_{1}$ animals from this line and crossed them to our previously characterized Afp::GFP reporter line (Kwon et al., 2006) to compare the level of reporter expression and the extent of its localization. Double hemizygous transgenic embryos were analyzed for fluorescent colocalization from early post-implantation stages to newborn pups.

\section{Comparative reporter expression in Afp::mCherrytg/+; Afp::GFPtg/+ embryos}

In agreement with our characterization of the Afp::GFP reporter (Kwon et al., 2006), mCherry was not detected at preimplantation stages. To perform a detailed characterization of the Afp::mCherry reporter, we determined the expression of mCherry by comparing it to GFP expression in double transgenic Afp::mCherry ${ }^{t g /+}$; Afp ::GFP ${ }^{t g /+}$ embryos. Red fluorescence was first detected specifically in the visceral endoderm at pre-streak (PS) stages, and appeared concomitantly with GFP-positive cells (Fig. 2a). Robust and 
homogenous mCherry reporter expression was maintained within the visceral endoderm through to the early streak (ES) stage (E6.5) (Fig. 2b). We noted that at these early postimplantation stages, mCherry-positive, as well as GFP-positive, cells encompassed the entire visceral endoderm, evident by homogenous fluorescence on the surface of the embryo; the only exception being the most proximal regions of the exVE. The exVE overlying the extraembryonic ectoderm was devoid of mCherry expression adjacent to the ectoplacental cone. Absence of transgene expression from this most proximal portion of the exVE was expected, and is due to signals originating from the extraembryonic ectoderm that repress Afp transcription until mid streak stages (MS) when extraembryonic mesoderm emerges and in doing so alleviates this repression (Dziadek, 1978; Dziadek and Adamson, 1978; Kwon et al., 2006).

Our previous analysis of Afp::GFP embryos revealed that around the early to late bud (EBLB) stages, GFP-positive emVE cells persisted in association with the epiblast (Kwon et al., 2008). These emVE-derived cells were detected because they exhibited perdurance of the GFP protein. Wholemount in situ hybridization experiments revealed that GFP transcripts were no longer being synthesized in the emVE at ES stages onwards. In agreement with these observations we observed cells that were both GFP-positive and mCherry-positive overlying the epiblast in Afp::mCherry ${ }^{t g /+} ; A f p:: G F P^{t g /+}$ embryos (Fig. 2c-g).

At the EB stage, a time when emVE cells are being dispersed by the intercalation of epiblast-derived definitive endoderm cells, we noted that all dispersing GFP-positive cells overlying the epiblast were also mCherry-positive (Fig. 2c-d). At this stage the localization of mCherry exhibited an almost complete overlap with the localization of GFP. $m R N A$ in situ hybridization indicated that while at the ES stage $m$ Cherry and GFP transcripts encompassed the entire VE, at the LB stage transcripts for both fluorescent proteins were only present in the exVE (Fig. 2h-i). Therefore, we could conclude that emVE cells that were mCherry-positive were being labeled due to the perdurance of the reporter protein.

The visualization of GFP-positive emVE derivatives overlying the epiblast in Afp::GFPtg/+ embryos suggested that these derivatives of the emVE contribute to, and are incorporated within, the gut tube of the embryo (Kwon et al., 2008). By revealing a lineage relationship between the visceral endoderm and the gut endoderm of the embryo, these data challenge the strict segregation of embryonic (epiblast) and extraembryonic (primitive endoderm) lineages (Rossant and Tam, 2009). Notably, these conclusions are now further supported by our observations in Afp::mCherry ${ }^{t g /+}$ embryos.

By the LB stage, when emVE cell dispersal was complete, both fluorescent proteins generally co-localized, such that majority of VE cells were GFP-positive; mCherry-positive. However, we also identified a small population of GFP-positive; mCherry-low cells, as well as some GFP-positive; mCherry-negative cells (Fig. 2e-g). Notably, we failed to detect any GFP-negative; mCherry-positive cells. We attributed this to the increased levels of fluorescence of GFP compared to those of mCherry, such that the latter reporter protein perdurance was not as extended. A detailed analysis of the two fluorescent reporters in visceral endoderm cells in PS to 5-somite stage embryos indicated a gradually increasing disparity between the numbers of cells expressing the GFP and mCherry reporters (Fig. 3c). This was accompanied with an increasing number of mCherry expressing cells that exhibited reduced levels of fluorescence. Nonetheless, the Afp::mCherry line serves as an attractive spectrally-distinct alternative to the $A f p:: G F P$ line for combinatorial short-term live imaging of the visceral endoderm lineage.

By early somite stages, red and green fluorescence was noted as co-localizing in the yolk sac of Afp::mCherry ${ }^{t g /+}$; Afp::GFP $P^{t g /+}$ embryos, as well as in the region of the posterior visceral 
endoderm overlying the primitive streak, in the crown cells around the node, and in cells located on either side of the anterior midline. These data support our previous observations (Kwon et al., 2008), by revealing a distinct VE-delineated separation between midline tissues, which will form the notochord, and the more laterally located gut endoderm, which will form the future gut tube (Fig. 3). We noted that the majority of GFP-positive emVEderived cells positioned laterally overlying the epiblast, within the gut endoderm, were also mCherry-positive, yet a subset exhibited diminished (low) or no red fluorescence (Fig. 3c).

\section{Additional sites of $\boldsymbol{m}$ Cherry reporter expression during organogenesis}

At around the 12 somite stage ( E8.75), the embryo initiates a process of turning, where it progresses from a lordotic to a fetal position, and in doing so internalizes the emVE containing gut endoderm to form the gut tube. At this stage exVE derivatives represent the endoderm layer of the visceral yolk sac.

E9.0 Afp::mCherry ${ }^{t g /+} ;$ Afp ::GFP $P^{t g /+}$ embryos expressed high levels of both mCherry and GFP in the yolk sac (Fig. 4a). Wholemount imaging of the embryo-proper isolated from the intensely fluorescent yolk sac enabled detection of both red and green fluorescence along the entire gut tube (Fig. 4b). Sections through the yolk sac confirmed that reporter fluorescence was restricted to the endoderm (Fig. 4c).

We previously noted GFP-positive cells along the gut tube of $A f p:: G F P^{t g /+}$ embryos, and suggested that they represented emVE-derived cells exhibiting perdurance of GFP, since no de novo transcription of the transgene occurs at this stage (Kwon et al., 2008). Observations made in Afp::mCherry ${ }^{t g /+}$; Afp ::GFP ${ }^{t g /+}$ embryos supported these previous studies. We noted GFP-positive; mCherry-positive cells in the epithelium of the gut tube throughout its anterior-posterior length; including the foregut, midgut and hindgut (Fig. 4d-f). In the majority of cases, levels of red fluorescence were low, further supporting our interpretation that mCherry protein exhibited reduced perdurance and therefore, in the absence of transcription, was visible for a shorter period of time, than GFP protein.

By E12.5 we observed mCherry-positive cells throughout the gastrointestinal tract and its associated organs in addition to the yolk sac endoderm of Afp::mCherry ${ }^{t g /+}$; Afp ::GFP $P^{t g /+}$ embryos (Fig. 5). As at earlier stages, mCherry-positive cells were always GFP-positive. mCherry-positive cells were found along the different regions of the gut tube, and gut endoderm-derived organs such as the developing pancreas, as well as in the majority of GFP-positive cells of the endoderm of the visceral yolk sac. At these stages, cells in the liver were mosaically labeled with GFP and mCherry. Interestingly, this was the only site at which we detected cells that were mCherry-positive; GFP-negative (Fig. 5e-f).

The same enhancer/promoter cis-regulatory sequence from the $A f p$ locus was used to generate both the Afp::mCherry and the Afp::GFP constructs. Thus, in principle, the two fluorescent protein reporters should exhibit complete concordance, except perhaps in tissues in which reporter visualization is due to fluorescent protein perdurance, or where reporter expression fluctuates within a population of cells. Alternatively, the disparity in reporter expression observed in the liver might be due to different insertion sites of the two transgenes, causing minor variations in expression dynamics in a subset of tissues. Even so, collectively these data suggest that the Afp::mCherry reporter faithfully recapitulates the Afp::GFP reporter expression at mid-gestation stages. And as with the Afp::GFP transgene, Afp::mCherry is likely to be de novo activated at several sites during these later embryonic stages. 


\section{Expression of mCherry in newborn Afp::mCherry mice}

To determine the sites of expression of the Afp::mCherry reporter at postnatal stages, we analyzed newborn animals at postnatal day (P) 3. At P3 several organs of Afp::mCherry ${ }^{t g /+}$; Afp::GFPtg/+ mice exhibited robust red (as well as green) fluorescence (Fig. 6). We detected cells in the brain and stomach positive for both GFP and mCherry (Fig 6a,b). Wholemount and section views of the liver revealed that nearly all cells exhibited red fluorescence (Fig. $\left.6 c-c^{\prime \prime}\right)$. Localization of the mCherry reporter was also noted in the pancreas (Fig. 6d,d'), and along the intestinal villi (Fig. 6e-e").

In summary, we have generated and characterized an Afp::mCherry transgenic line. Afp::mCherry is a novel red fluorescent reporter line that provides labeling throughout the $\mathrm{VE}$ and its derivatives in the early embryo. The Afp::mCherry line is comparable to our previously reported Afp::GFP line (Kwon et al., 2006; Kwon et al., 2008). We expect that Afp::mCherry will be useful as a spectrally-distinct widespread VE reporter between PS to LS stages, and as a short-term VE lineage tracer between EB and 15 somite stages. In addition, it can also serve as a fluorescent reporter in several Afp expressing tissues during normal and perturbed embryonic development, as well as during homeostasis and disease progression in adult mice.

\section{METHODS}

\section{Transgenic construct generation, cell line transfection, and mouse strain establishment}

The pAfp::mCherry plasmid was generated in two steps. First, as an intermediate, the plasmid $p C X:: m C h e r r y$ was generated by PCR of $m$ Cherry from $p R S E T m C h e r r y$ (Shaner $e t$ al., 2004) using primers $5^{\prime}-$

CCGGAATTCCGGACGCGTCGACGTCGATGGTGAGCAAGGGCGAGGAGGATAAC ATG-3' and 5'- CCGGAATTCCGGTTACTTGTACAGCTCGTCCATGCCGCCGGT-3' containing EcoRI and SalI restriction sites and to clone into the EcoRI site of $p C A G G S$ (Niwa et al., 1991). Then, $p C X:: m$ Cherry was used as a template to obtain a $m C h e r r y$ Rabbit-globin-PolyA DNA fragment by PCR using primers 5'CCGGAATTCCGGACGCGTCGACGTCGATGGTGAGCAAGGGCGAGGAGGATAAC ATG-3' and 5'GCGGCCGCTATGGCCTTTTGACGTCGACGCGTGGGCTGCAGGTCGAGGGATCTC CA-3'. The resulting PCR product was then cloned into the SalI site of $p S P 73:: A f p$ (Hammer et al., 1987; Spear and Tilghman, 1990) to obtain pAfp::mCherry.

HepG2, Caco2 and Hepa1-6 (ATCC CRL1830) cells were transfected with Lipofectamine 2000 reagent (Invitrogen, CA) according to manufacturer's recommendations, and imaged for fluorescence within $24-48$ hours.

To generate the Afp::mCherry transgenic mouse line, $p A f p:: m$ Cherry was linearized with XhoI, purified using standard protocols (Nagy A, 2003), and micro-injected into C57BL/6 zygotes by the Memorial Sloan-Kettering Cancer Center Transgenic Core Facility. Founder $\left(\mathrm{F}_{0}\right)$ animals were identified by Southern blot hybridization and PCR.

\section{Mouse husbandry}

Three $\mathrm{F}_{0}$ animals that were noted as positive by Southern blot and PCR genotyping for $m$ Cherry were screened for localization of red fluorescence in postimplantation embryos by crossing to ICR animals. Upon identification of the most robustly and uniformly expressing founders, $F_{1}$ animals were recovered by crossing to ICR animals, which were then backcrossed and maintained on an ICR background. All analyses presented are of hemizygous (tg/+) transgenic animals. For co-localization studies with the Afp::GFP 
reporter (Kwon et al 2006), mice were crossed to the Afp::GFP strain to generate double hemizygous transgenics.

\section{Embryo dissection and processing}

Embryos were dissected in DMEM-F12 media containing 5\% newborn calf serum, fixed at $4^{\circ} \mathrm{C}$ over night in $4 \%$ paraformaldehyde in phosphate-buffered saline (PBS), then washed three times in PBS. Post-implantation embryos were staged according to Downs and Davies, 1993. For embryos older than E12.5 and including postnatal and adult animals, the organs were extracted by manual dissection in PBS and then processed as above. Fixed embryos and organs were taken through a sucrose gradient, embedded in OCT (TissueTek compound) and cryosectioned at $12 \mu \mathrm{m}$.

\section{In situ hybridization and immunochemistry}

In situ hybridization to wholemount embryos was performed according to standard protocols (Nagy A, 2003). The $m$ Cherry antisense probe was generated using a pBluescript $S K+$ plasmid containing the $m$ Cherry cDNA. To detect nuclei, wholemount or cryosectioned samples were counterstained with a 1:1000 PBS dilution of Hoechst (Invitrogen, Carlsbad, CA).

\section{Image data acquisition and processing}

Widefield images were acquired with a Zeiss Axiocam MRc or MRm CCD camera mounted on a Leica MZ165FC stereo-dissecting microscope. Laser scanning confocal data was acquired with a Zeiss LSM510 META mounted on a Zeiss Axiovert 200M microscope. Fluorophores were excited using a $405 \mathrm{~nm}$ diode laser (Hoechst), $488 \mathrm{~nm}$ argon laser (GFP), or $543 \mathrm{~nm}$ HeNe laser (mCherry). The objectives used were Plan-Apo 20x/NA0.75 and Fluar 5x/NA0.25. Wholemount embryos were imaged in MatTek dishes (Ashland, MA). Cryosectioned samples were mounted onto glass slides and imaged through glass coverslips. Confocal images were acquired as $z$ stacks of $x-y$ images taken at $0.2-2 \mu \mathrm{m} z$ intervals. Raw data was processed using Zeiss AIM software (Carl Zeiss Microsystems, http://www.zeiss.com) or Volocity (Perkin-Elmer, http://www.cellularimaging.com/products/volocity/). Figures were assembled and annotated in Adobe Photoshop CS4.

\section{Acknowledgments}

We thank the MSKCC Mouse Genetics Core Facility for production of transgenic founders; Roger Tsien for the pRSETmCherry plasmid; members of our lab for discussions, and Maria Pulina for comments on the manuscript. Work in our laboratory is supported by the NIH (RO1-HD052115 and RO1-DK084391) and NYSTEM. MV was supported by a Sloan-Kettering Division (SKD) Frank Lappin Horsfall graduate student fellowship. SN was supported by an American Heart Association (AHA) postdoctoral fellowship.

\section{LITERATURE CITED}

Campbell RE, Tour O, Palmer AE, Steinbach PA, Baird GS, Zacharias DA, Tsien RY. A monomeric red fluorescent protein. Proc Natl Acad Sci USA. 2002; 99:7877-7882. [PubMed: 12060735]

Downs KM, Davies T. Staging of gastrulating mouse embryos by morphological landmarks in the dissecting microscope. Development. 1993; 118:1255-1266. [PubMed: 8269852]

Dziadek M. Modulation of alphafetoprotein synthesis in the early postimplantation mouse embryo. $\mathrm{J}$ Embryol Exp Morphol. 1978; 46:135-146. [PubMed: 81255]

Dziadek M, Adamson E. Localization and synthesis of alphafoetoprotein in post-implantation mouse embryos. J Embryol Exp Morphol. 1978; 43:289-313. [PubMed: 75937] 
Ferrer-Vaquer A, Viotti M, Hadjantonakis AK. Transitions between epithelial and mesenchymal states and the morphogenesis of the early mouse embryo. Cell Adh Migr. 2010; 4:447-457. [PubMed: 20200481]

Fink D, Wohrer S, Pfeffer M, Tombe T, Ong CJ, Sorensen PH. Ubiquitous expression of the monmeric red fluorescent protein mCherry in transgenic mice. Genesis. 2010

Hammer RE, Krumlauf R, Camper SA, Brinster RL, Tilghman SM. Diversity of alpha-fetoprotein gene expression in mice is generated by a combination of separate enhancer elements. Science. 1987; 235:53-58. [PubMed: 2432657]

Kwon GS, Fraser ST, Eakin GS, Mangano M, Isern J, Sahr KE, Hadjantonakis AK, Baron MH. $\mathrm{Tg}$ (Afp-GFP) expression marks primitive and definitive endoderm lineages during mouse development. Dev Dyn. 2006; 235:2549-2558. [PubMed: 16708394]

Kwon GS, Hadjantonakis AK. Transthyretin mouse transgenes direct RFP expression or Cre-mediated recombination throughout the visceral endoderm. Genesis. 2009; 47:447-455. [PubMed: 19415627]

Kwon GS, Viotti M, Hadjantonakis AK. The endoderm of the mouse embryo arises by dynamic widespread intercalation of embryonic and extraembryonic lineages. Dev Cell. 2008; 15:509-520. [PubMed: 18854136]

Mesnard D, Guzman-Ayala M, Constam DB. Nodal specifies embryonic visceral endoderm and sustains pluripotent cells in the epiblast before overt axial patterning. Development. 2006; 133:2497-2505. [PubMed: 16728477]

Nagy, A.; G, M.; Vintersten, K.; Behringer, R. Manipulating the mouse embryo: A laboratory manual. 3rd ed.. Cold Spring Harbor Laboratory Press; Plainview, NY: 2003.

Niwa H, Yamamura K, Miyazaki J. Efficient selection for high-expression transfectants with a novel eukaryotic vector. Gene. 1991; 108:193-199. [PubMed: 1660837]

Nowotschin S, Eakin GS, Hadjantonakis AK. Dual transgene strategy for live visualization of chromatin and plasma membrane dynamics in murine embryonic stem cells and embryonic tissues. Genesis. 2009a; 47:330-336. [PubMed: 19358158]

Nowotschin S, Eakin GS, Hadjantonakis AK. Live-imaging fluorescent proteins in mouse embryos: multi-dimensional, multi-spectral perspectives. Trends Biotechnol. 2009b; 27:266-276. [PubMed: 19339068]

Nowotschin S, Hadjantonakis AK. Cellular dynamics in the early mouse embryo: from axis formation to gastrulation. Curr Opin Genet Dev. 2010; 20:420-427. [PubMed: 20566281]

Rossant J, Tam PP. Blastocyst lineage formtion, early embryonic asymmetries and axis patterning in the mouse. Development. 2009; 136:701-713. [PubMed: 19201946]

Shaner NC, Campbell RE, Steinbach PA, Giepmans BN, Palmer AE, Tsien RY. Improved monomeric red, orange and yellow fluorescent proteins derived from Discosoma sp. red fluorescent protein. Nat Biotechnol. 2004; 22:1567-1572. [PubMed: 15558047]

Spear BT, Tilghman SM. Role of alpha-fetoprotein regulatory elements in transcriptional activation in transient heterokaryons. Mol Cell Biol. 1990; 10:5047-5054. [PubMed: 1697927]

Srinivas S. The anterior visceral endoderm-turning heads. Genesis. 2006; 44:565-572. [PubMed: 17078044] 

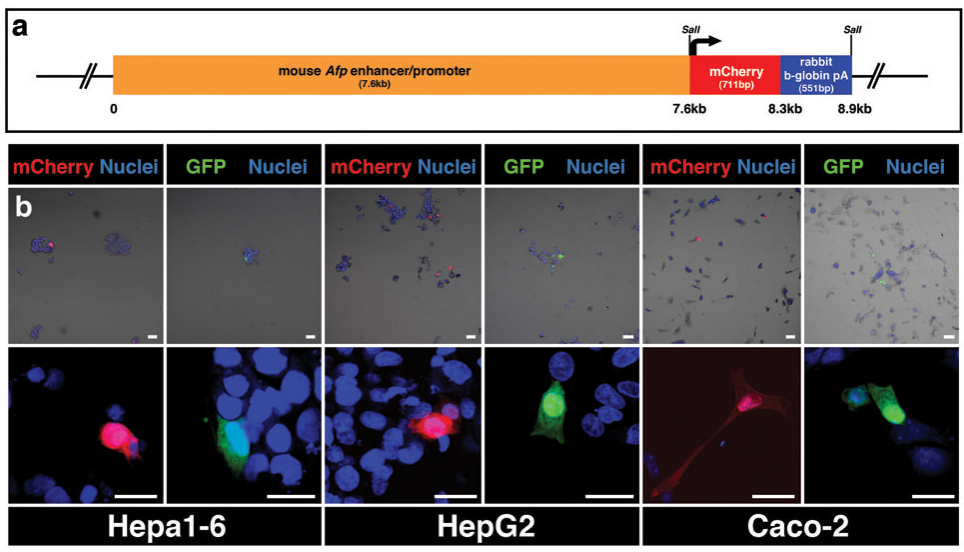

FIG. 1. Design and validation of the Afp::mCherry transgene in cells

(a) Schematic representation of the Afp::mCherry construct. (b) Transfection of the $p A f p:: m C h e r r y$ plasmid in cells expressing Afp endogenously resulted in expression of the mCherry reporter. Upper row depicts low magnification views of fluorescence overlayed onto the brightfield channel, lower row depicts high magnifications views of fluorescent channel. Hepa1-6, HepG2, and Caco-2 cells transfected with the plasmids pAfp::mCherry and Afp::GFP contained both red and green fluorescent cells. Cells were counterstained with Hoechst (blue). Scale bars represent $20 \mu \mathrm{m}$. 

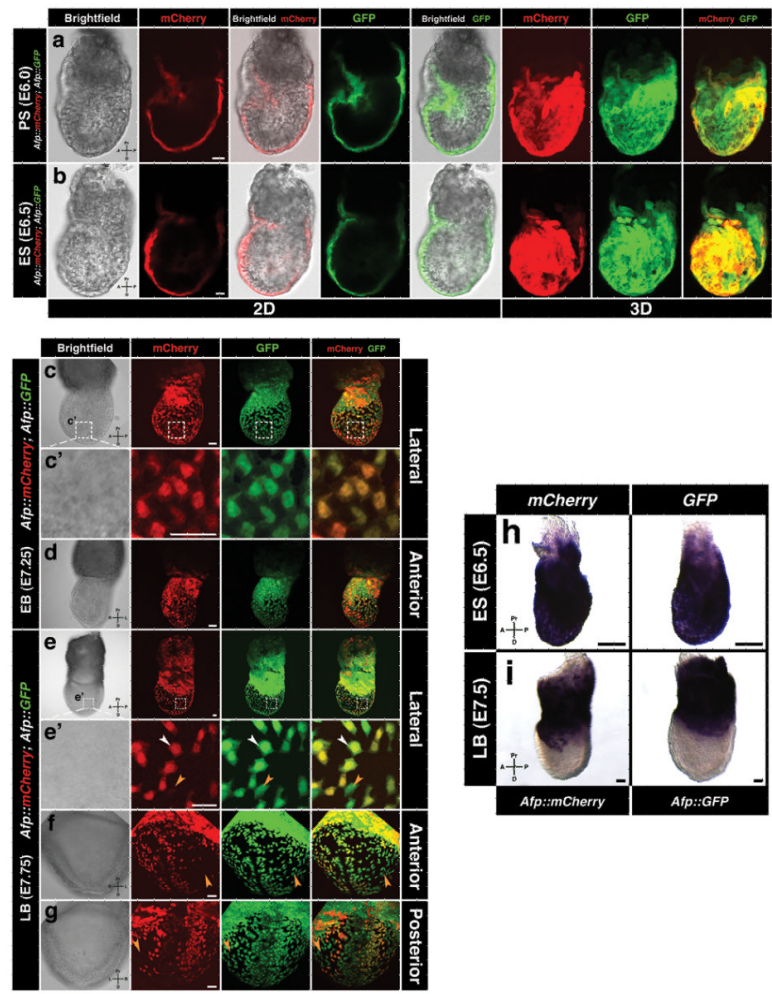

FIG. 2. Afp::mCherry transgene activity is detected throughout the visceral endoderm and its derivatives

(a) In PS stage (E6.0) Afp::mCherry ${ }^{t g /+}$; Afp::GFPtg/+ embryos, mCherry co-localized with GFP and was expressed throughout the VE with somewhat heterogenous intensities (both in emVE and exVE, except the most proximal part adjacent to the ectoplacental cone, where both fluorescent proteins are absent). (b) The overall correlation of mCherry and GFP expression was maintained at the ES (E6.5) stage. (c) Lateral view of an EB (E7.25) Afp::mCherry ${ }^{t g /+} ;$ Afp::GFPtg/+ embryo depicting mCherry-positive emVE cells and uniform fluorescence in the exVE, with identical GFP localization. (c') High magnification of early dispersal stage of VE, showing faithful co-expression of red and green fluorescence. (d) Anterior view depicting co-localization along the anterior midline. (e) Lateral view of LB (E7.75) stage Afp::mCherry ${ }^{t g /+} ;$ Afp::GFPtg/+ embryo showing red fluorescence in the exVE and dispersed emVE. ( $\left.\mathbf{e}^{\prime}\right)$ High magnification view of late dispersal stage of VE indicating co-localization of red and green fluorescence in the majority of cells (white arrowheads) and a subset of GFP-positive cells with low levels of mCherry (orange arrowheads). (f,g) Anterior and posterior views of the distal section of embryo overlying the epiblast indicating weaker or absence of red fluorescence in a subset of GFP-positive cells (orange arrowheads). (h) Whole-mount in situ hybridization in ES stage (E6.5) Afp::mCherry ${ }^{t g /+}$ embryos revealed $m$ Cherry transcript present throughout VE (both emVE and exVE), in agreement with the localization of GFP transcripts in stage-matched Afp::GFPtg/+ embryos. (i) By the LB stage (E7.5) mCherry transcripts appeared restricted to the exVE in Afp::mCherry ${ }^{\text {tg/+ }}$ embryos, again corresponding to the expression pattern of GFP in Afp::GFP ${ }^{\text {tg/+ }}$ embryos. Pr, proximal; D, distal; A, anterior; P, posterior; 2D, 2dimensions; 3D, 3-dimensions. Scale bars represent $20 \mu \mathrm{m}$ in a and b and $50 \mu \mathrm{m}$ in c-i. 


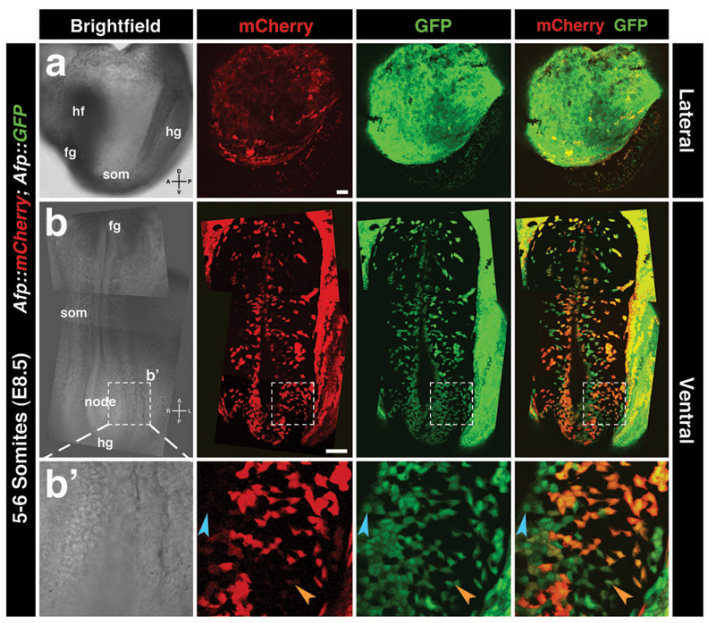

C

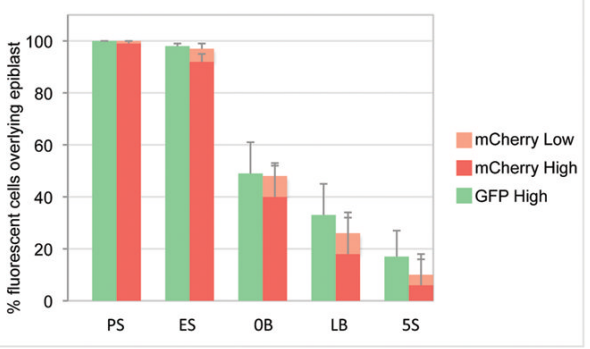

FIG. 3. Organization of mCherry-positive cells around the node and notochordal plate (a) Lateral view of an Afp::mCherry ${ }^{t g /+} ; A f p:: G F P^{t g /+}$ embryo at the 5-6 somite stage (E8.5) showing widespread red fluorescence in the visceral yolk sac. (b) Ventral view revealing stereotypical organization of mCherry-positive cells around the node and bilaterally along the notochordal plate. ( $\left.\mathbf{b}^{\prime}\right)$ High magnification view indicating absence (blue arrowheads) or low levels (orange arrowheads) of mCherry in a subset $(\sim 50 \%)$ (percentage adjusted after quantitation) of GFP-positive cells. (c) Histogram (mean $\pm \mathrm{sd}$ ) depicting percent fluorescent cells overlying the epiblast. Fluorescence was measured using Volocity software, where 'high' fluorescence was determined as above fluorescent intensity of 50, and 'low' fluorescence was considered in the range between intensity of 50 and background levels. $\mathrm{N}=3$ embryos for each stage. Pr, proximal; D, distal; A, anterior; P, posterior; fg, foregut invagination; som, somites; hg, hindgut invagination. Scale bars represent $100 \mu \mathrm{m}$. 


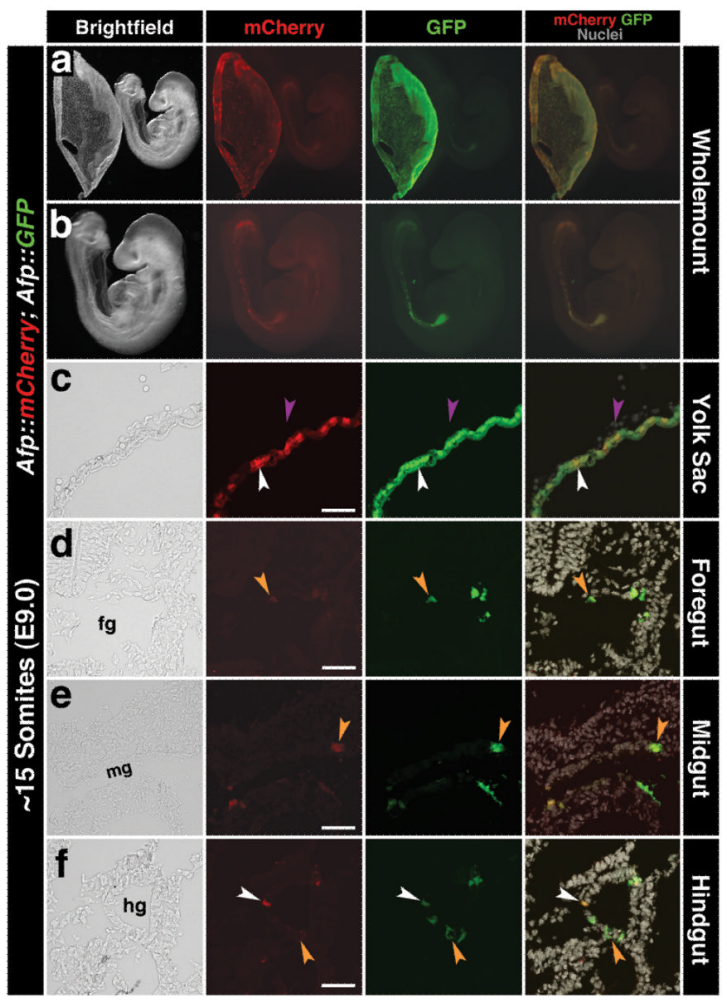

FIG. 4. Afp::mCherry expression in the $\sim 15$ somite stage embryo

(a) Lateral wholemount view of Afp::mCherry ${ }^{t g /+}$; Afp::GFP ${ }^{t g /+}$ E9.0 embryo illustrating widespread red and green fluorescence in the yolk sac. (b) View of the same embryo but in isolation from the yolk sac, allowing detection of green and red fluorescence along the gut tube. (c) Section through the yolk sac indicating localization of mCherry specifically in the endoderm layer. Cells of the endoderm were mostly positive for red and green fluorescence (white arrowheads) while yolk sac mesoderm cells of the yolk sac were non-fluorescent (purple arrowheads). (d-e) Sections through foregut and midgut regions revealed cells in the endodermal lining of the gut tube lumen being positive for GFP with low levels of mCherry (orange arrowheads). (f) In hindgut sections some endoderm cells were positive for both fluorescent proteins (white arrowheads), while other cells were GFP-positive, with low or undetectable levels of mCherry (orange arrowheads). fg, foregut invagination; som, somites; hg, hindgut invagination. Scale bars represent $50 \mu \mathrm{m}$. 


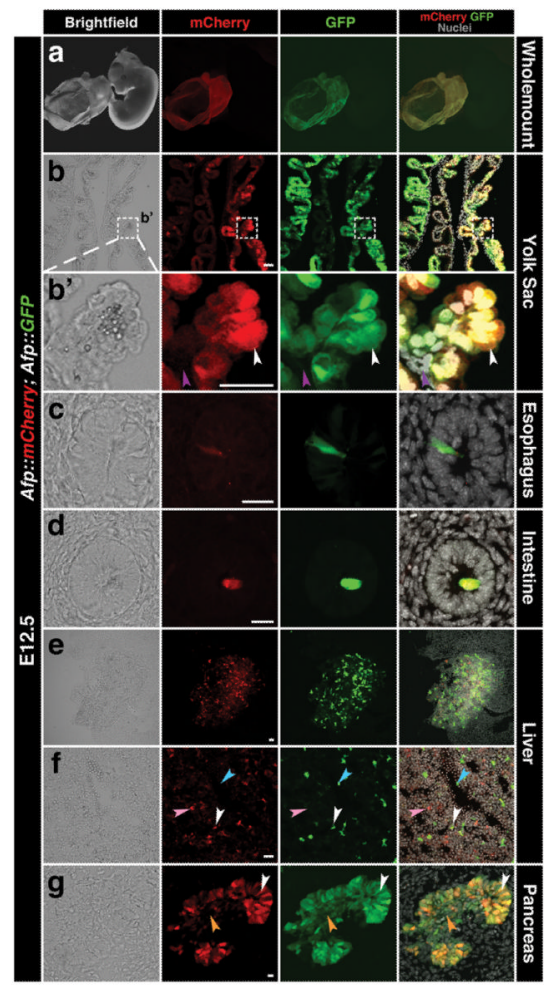

FIG. 5. Additional sites of mCherry localization during organogenesis

(a) Lateral wholemount view of an Afp::mCherry ${ }^{t g} /+$; Afp::GFPtg/+ embryo at E12.5 indicating red and green fluorescent reporter expression in the yolk sac. (b-g) Sections taken through the embryo in (a) revealing Afp::mCherry transgene activity in endoderm derivatives. (b) Section through the yolk sac revealed that the majority of GFP-positive cells were also mCherry-positive. ( $\mathbf{b}^{\prime}$ ) High magnification of the section of yolk sac indicating that fluorescence is specific to the endoderm layer. Fluorescent cells (white arrowheads) form a contiguous epithelium distinct from a second, non-fluorescent layer (purple arrowheads). (c,d) Sections of gut tube derivatives with double fluorescent cells. (e,f) The

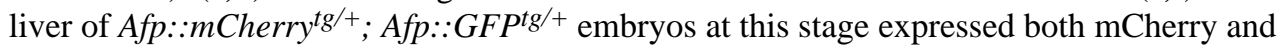
GFP, yet these reporters did not necessarily co-localize at a cellular level. Some liver cells were non-fluorescent, some were only mCherry-positive (pink arrowheads), some were only GFP-positive (blue arrowhead) while others were positive for both reporters (white arrowheads). (g) Section of the pancreatic bud reveals that all cells were GFP-positive, a large number were also mCherry-positive (white arrowheads) and in a subset of cells mCherry was either present at low levels or absent (orange arrowheads). Scale bars represent $100 \mu \mathrm{m}$. 

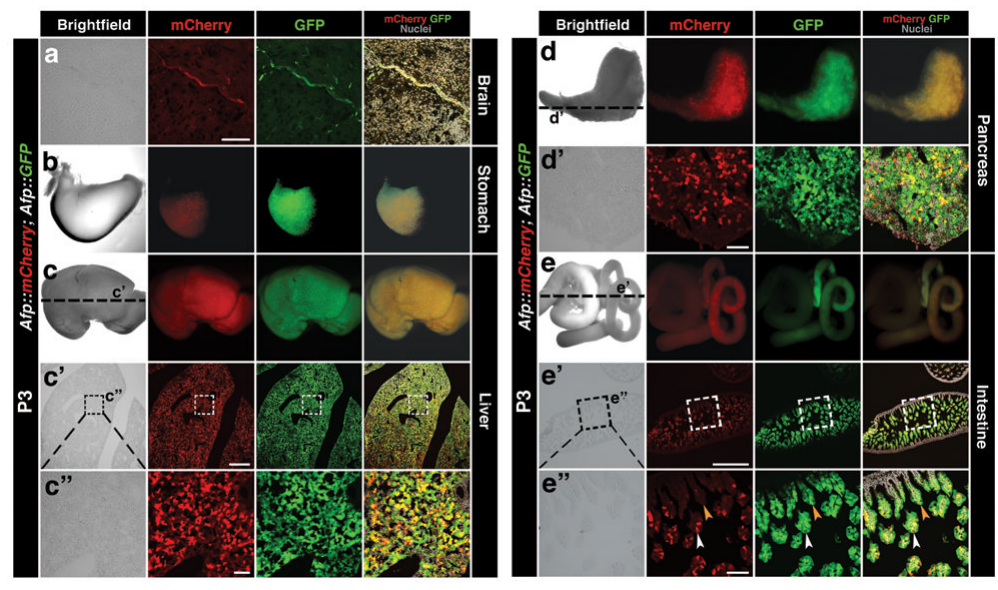

FIG. 6. Localization of mCherry in organs of newborn mice

(a) Section through an Afp::mCherry ${ }^{t g /+}$; Afp ::GFP ${ }^{t g /+} \mathrm{P} 3$ brain revealing cells positive for both fluorescent protein reporters. (b) Wholemount view of the newborn stomach revealing red fluorescence in GFP-positive cells. (c) Wholemount image of the liver indicating widespread fluorescence of both GFP and mCherry. ( $\left.\mathbf{c}^{\prime}-\mathbf{c}^{\prime \prime}\right)$ Sections through the liver depicted in (c) revealed extensive, but not always correlative reporter expression. (d) Wholemount view of the newborn pancreas showing broad coincidence of GFP and mCherry. ( $\mathbf{d}^{\prime}$ ) Section through the pancreas indicating mCherry fluorescence in the majority of GFP-positive cells. (e) Wholemount view of the newborn intestine indicating extensive regions of reporter co-localization. ( $\left.\mathbf{e}^{\prime}-\mathbf{e}^{\prime \prime}\right)$ Sections through the intestine revealing cells along the villi positive for both GFP and mCherry (white arrowheads), or GFP-positive with low or undetectable levels of mCherry (orange arrowheads). Scale bars represent $500 \mu \mathrm{m}$ in $\mathrm{c}^{\prime}$ and $\mathrm{e}^{\prime}$, and $100 \mu \mathrm{m}$ in all other panels. 